\title{
An Efficient Medical Image Fusion Technique based on IT2FLDS
}

\author{
Ramya H. R. \\ Research Scholar M S R I T \\ Bengaluru-560054
}

\author{
B. K. Sujatha \\ Professor M S R IT \\ Bengaluru-560054
}

\begin{abstract}
Multiple modal medical image fusion is an essential method for medical imaging technologies. In these Multi-modal medical image fusion usually are Positron Emission Tomography(PET)) and Single-photon Emission Computer Tomography(SPECT), Magnetic Resonance Imaging (MRI) and Computed Tomography (CT) images are utilized. However, the conventional state-of-art-fusion-techniques consists of less redundant and less comprehensive information. Therefore, here, we present an image fusion technique to control non-linear uncertainties and provide stability based on IT2FLDS for multi-modal medical color images. The core idea is to perform fusion on color source images of either functional or structural type by extracting both large and small structural information which is rarely done in any other conventional state-of-art-techniques. This can be achieved with the help of IT2FLDS. The fuzzy membership functions based image fusion technique helps to combining low and high frequency components of multimodel medical color images. Experimental results proves the superiority of our proposed image fusion model in terms of visual and quantitative analysis like mutual information, standard deviation and Feature Point Similarity Measure.
\end{abstract}

\section{Keywords}

Multi-modal; Magnetic Resonance Imaging; Image fusion; type-2 fuzzy logic

\section{INTRODUCTION}

In recent time, multi-image sensors are extensively deployed to help the various visual devices under different environmental circumstances. Moreover, various innovated medical imaging methods are becomes an essential tool to analyzing various diseases in very little time and these methods also helps to provide information for the effective diagnosis of those diseases which led to better diagnosis in a cost-effective manner. The availability of various bio-medical images helps in clinical diagnosis of different diseases. The medical imaging methods can be partitioned into two parts such as functional methods and structural methods [1]. For instance, the functional medical imaging methods are PET and SPECT whereas MRI and CT comes under structural medical imaging. Both imaging methods helps in the diagnosis of various diseases in patients [2]. However, it is very difficult for any single medical imaging modality to provide efficient diagnosis of these diseases due to extensive growth of medical imaging data [3-4].

Moreover, MRI and CT images can be widely used in various medical applications. The MRI image quality directly affects the diagnosis accuracy. MRI imaging modularity is a noninstructive method which is used to detect the internal organs and muscles of a patient. MRI and CT images both are mostly complimentary in nature. CT images helps to determine the extent of disease. Therefore, MRI and CT images both becomes essential parts of the modern medical applications which is used to provide structural and anatomical information. However, a single medical image can only provide some precise information, not all the necessary information and not able to handle the extensive growth of medical data. Therefore, the image fusion process can be highly effective with all the required information. Image fusion is a technique which can be used to fuse complementary images, multiple temporal images and multifocal images into one medical image with all the necessary required data from input medical images. The focal point of image fusion technique is to make appropriate composite image for Human Visual Systems (HVS). Moreover, these composite images can be further used for image processing applications, segmentation, visual image enhancement, denoising, feature extraction, object detection and image classification [5-6].This image fusion technique can be utilized in various fields for different disciplines such as image processing, videos surveillance, medical applications and remote sensing applications.

Consequently, the fused single image from two or more source images, consist of more inherent complementary information with appropriate visual quality and can be easily utilized for further processing [7]. Therefore, the multimodularity image fusion technique can play a vital role in image diagnosis, precise analysis and treatment preparation for medical applications due to its appropriate anatomical and physiological capabilities [8]. The medical images which need to be fused, can be of same or different modularity. Moreover, the fused medical images must contain high visual quality and must provide salient information from the source images and reduce inconvenient information and should remove artifacts from the images. However, even after enormous amount of success in medical image fusion technologies, few challenges also exists such as conventional state-of-art-techniques only concentrate on large structure details and they neglect small scale structural information. Hence, they often consist of noise in the fused images. Moreover, inadequate fusion rule is a big problem which evaluates the preciseness of complementary data present in the multimodal fused images. For instance, the well-known Average-Maximum rule can give darkened fusion results which are not appropriate [9-10].

Therefore, these challenges such as inefficient feature extraction, noise intolerance and inadequate fusion of complimentary information need to be focused soon to enhance the performance of fusion techniques [11]. Thus, to handle massive amount of medical data and to provide clinical analysis of various diseases, the advanced Decision Support System (DSS) is utilized. The advanced Decision Support System can access extensive medical data and can support in clinical diagnosis of diseases [12]. This DSS helps to observer huge amount of medical data in a much quicker time and in much detailed way [13]. DSSs usually get knowledge by utilizing information of real test cases whose classification is already done and this knowledge can helps to form new models which can efficiently classify these enormous medical data [14]. However, there are various complex uncertainties 
which are difficult to handle using Decision Support System (DSS) which can degrade the performance of diagnosis. In these type of cases, Fuzzy logics are widely adopted to counter these type of classification issues and to overcome from modeling issues in various application fields [15].

Moreover, fuzzy logics helps to enhance the efficiency of classification and Decision Support System (DSS) by overlapping some class definitions and also enhances the outcome interpretability by providing the access to internal structure of classifiers and decision making procedures [16] , [17]. Fuzzy logics can be portioned into two types such asT1FLS and IT2FLDS.Furthermore,T1FLS are utilized for the classification of various signals such as Normal Sinus Rhythm (NSR), Ventricular Tachycardia VT and Ventricular Fibrillation VF. However, the handling capabilities of uncertainties is bounded using T1FLS due to crisp membership functions of T1FLS and fuzzy set representation in T1FLS depends on the membership grades which are inadequate. Therefore, IT2FLDS are utilized over T1FLS which is an extension of T1FLS. Furthermore, Type-2 membership functions provide permission to model different uncertainties, which is not properly handled using type-1 membership functions [18]. Therefore, the IT2FLDS can be very helpful in providing efficient classification and improve decision making during fusion of two or more source images.

Thus, multi-model image fusion methods can provide efficient anatomical and physiological characteristics using IT2FLDS which helps in clinical diagnosis of images. Therefore, here, we present an image fusion technique to control non-linear uncertainties and provide stability based on IT2FLDS for multi-modal medical color images. This image fusion technique helps in clinical diagnosis of various diseases. The focal point is to provide efficient fusion technique on color input images of either functional or structural type by extracting both large and small structural information which is rarely done in any other conventional state-of-art-techniques. This can be achieved with the help of IT2FLDS. The T2FUZZY membership functions based image fusion technique helps to combining low and high frequency components of multi-model medical color images. The proposed image fusion technique can extract detailed information from the CT, MRI, MR-T1, MR-T2 and PET color images.

Moreover, initially, we will proceed with different combinations of various medical color source images of various types. Then, in our proposed image fusion technique, the main idea is to convert the input color images from RGB color map to YUV color map. Then, both the achromatic channel $\mathrm{Y}$ of the input color image and input gray scale image is fused together using the proposed image fusion technique to get high intensity of a color image. Finally, the YUV color space is again converted into RGB color space. In this manner, a final fused image is attained with the help of our proposed image fusion technique as demonstrated in figure 1. The proposed model gives more contrast increment than any other state-of-art techniques due to its complete color utilization with the help of Opponent-process theory in human perception. This technique can help in providing proper treatment and clinical diagnosis in the field of medical applications. This technique gives precise and detailed information of various objects and reduces significant amount of memory by storing fused color image from multi-model source color images. Experimental results proves the superiority of our proposed image fusion model in terms of visual and quantitative analysis.
This paper is organize in following sections, which are as following. In segment 2, we defines about the image fusion issues and how they can be eliminated by using our proposed model. In section 3, we described our proposed methodology for color image fusion of various image classes. In section 4 , simulations outcomes and performance evaluation shown, and segment 5 summarizes our paper.

\section{LITERATURE SURVEY}

In recent time, a decent amount of work has been done in signal systems to increase the efficiency of image fusion schemes. The image fusion techniques can be extensively utilized in various fields such as image processing, videos surveillance, medical applications and remote sensing applications and healthcare solutions. Fuzzy logics can be utilized to improve quality and visual presentation of multimodel color images with the integration of image fusion techniques which combine complementary and redundant data of more than or exactly two color images in a single color image with detailed information. Moreover, IT2FLDS and MF helps to provide efficient decision making and can be utilized for preprocessing, survey processing, time-series forecasting and function approximation [19-21]. Here, an extensive literature is presented on image fusion techniques which are as follows:

In [22], a novel image fusion technique is employed based on Non-Subsampled Contourlet Transform (NSST) for CT and MRI medical images to reduce "Gibbs effect" problem which occurs is conventional state-of-art-technique and execution speed of processing. This technique helps to identify the efficient and detailed information of fusion image. This technique helps to fuse low and high frequency components to get better feature quality. However, an optimization problem may occur using this technique. In [23], a novel image fusion technique is employed based on Shearlet Transform Singular Value Decomposition (STSVD) and Shearlet Transform Discrete Cosine Transform (STDCT) for PET and MRI images to fuse low-pass and high-pass sub-band. This technique helps to retain data from both source color images. In [24], an image fusion technique is introduced based on bidimensional empirical mode decomposition (BEMD) and IHS methods to fuse MRI and PET images. In this technique, HIS method is utilized to decompose PET image and BEMD method is utilized to examine the PET image intensity. However, distortion may occur in visual quality of images. In [25], image fusion enhancing technique is introduced to analyze SPECT and MRI images. This fusion technique helps to extract efficient features and mutual information from the images. In [26], a discrete cosine transform (DWT) and Adaptive Histogram Equalization based fusion technique is presented for MRI and PET Images to retain efficient color and spectral information in source color images. The Adaptive Histogram Equalization method is utilized to attain less color distortion and high contrast output. In [27], a multi-model supervised fusion technique is introduced with reference model MCCAR_JICA to utilize prior information for MRI images. This technique gives stable results in different noise levels. This technique can be utilized to enhance inter-subject covariation and spatial independence between MRI image components. However, an optimization problem may occur using this technique In [28], a novel image fusion technique is introduced based on 2-D Hilbert transform and IHS method for MRI and PET images. This technique is introduced both spatial and spectral features from source color medical images. This technique provides better qualitative and quantitative also get analysis and quality of fused color 
images enhanced. In [29], a label fusion technique is introduced which rely upon sparse patch representation to fuse MRI images. This technique can be used to get efficient feature maps and to measure similarity and to evaluate label information to label fusion.

An extensive literature is presented on image fusion techniques and their performance and issues are discussed. In these conventional state-of-art-techniques, sensor uncertainties and noise can disturb the decision making abilities of the system and disability to preserve the original chromaticity issue is also very well-known. To reduce uncertainties and get efficient reconstructed medical color images and preserve original chromaticity, here, we have introduced an image fusion technique to provide stability based on IT2FLDS for multi-modal medical color images. The sensor data uncertainties can be controlled efficiently using Interval Type 2 Gaussian MF. Here, a Comprehensive explanation of on Type-2 Fuzzy Logic systems is presented and IT2FLS toolbox helps to build an efficient IT2FLDS.Experimental results verifies that IT2FLDS can control sensor uncertainties in much effective way than type- 1 fuzzy logics [30].

\section{Medical Image Fusion representation Using Interval Type-2 Fuzzy logics Decision System (IT2FLDS):}

Medical Image fusion technique extract and combines appropriate and detailed information from different modalities of source color images into a fused color image. Moreover, the resultant image consists of much detailed information in contrast to source images which are utilized in fusion process and resultant image is more suitable in terms of visual perception and feature points. The resultant image produced with the help of image fusion technique can be stored, which helps to reduce storage capacity by avoiding the multimodal input source images. This image fusion technique can be widely utilized in image processing, videos surveillance, medical applications, healthcare solutions, face recognition and remote sensing applications etc. However, the conventional state-of-art-techniques contains various issues like sensor uncertainties and noise can disturb the decision making abilities of the system, disability to preserve the original chromaticity issue and inappropriate feature extraction etc.

Therefore, here, we present an image fusion technique to control non-linear uncertainties and provide stability based on IT2FLDS for multi-modal medical color images. The proposed image fusion technique extract a detailed data from both input color images and fuse them into one color image with higher visual perception. IT2FLDS is used to attain efficient local features and determine each pixel weight. Experimental results proves the superiority of proposed fusion model in terms of visual and quantitative analysis. The wellknown benefit of IT2FLDS is its management of uncertainty and it works precisely even in the presence of noise. Here, figure 1 demonstrate the architectural diagram of the proposed medical fusion technique using IT2FLDS.

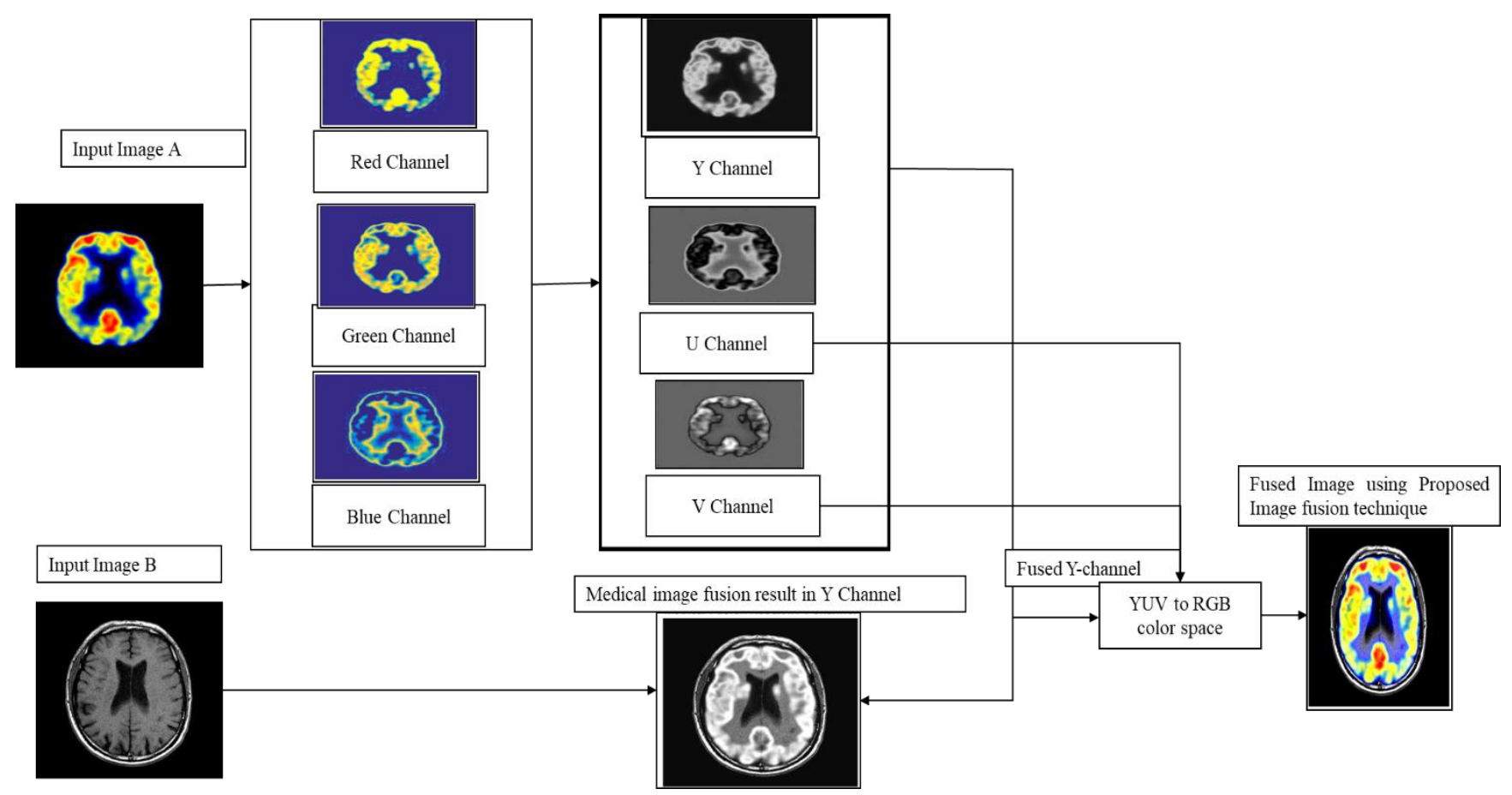

Figure 1 Block diagram of the proposed medical image fusion technique using interval type-2 fuzzy logic system

The proposed fusion method is utilized to decrease noise in source multimodal color medical images and fuse them into one color image with higher visual perception. Initially, we will proceed with different combinations of various medical color source images of various types. Then, in our proposed image fusion technique, the main idea is to convert the input color image from RGB color map to YUV color map. Then, both the achromatic channel $\mathrm{Y}$ of the input color image and input gray scale image is fused together using the proposed image fusion technique to get high intensity of a color image. Finally, the YUV color space is again converted into RGB color space. In this manner, a final fused image is attained with the help of our proposed image fusion technique as demonstrated in figure 1.The proposed fusion method gives more contrast increment than any other state-of-art techniques due to its complete color utilization with the help of Opponent-process theory in human perception. This technique can help in providing proper treatment and clinical diagnosis 
in the field of medical applications. This technique gives precise and detailed information of various objects and reduces significant amount of memory by storing fused color image from multi-model source color images. Here, figure 2 demonstrates the Schematic of proposed IT2FLDS for image fusion applications.

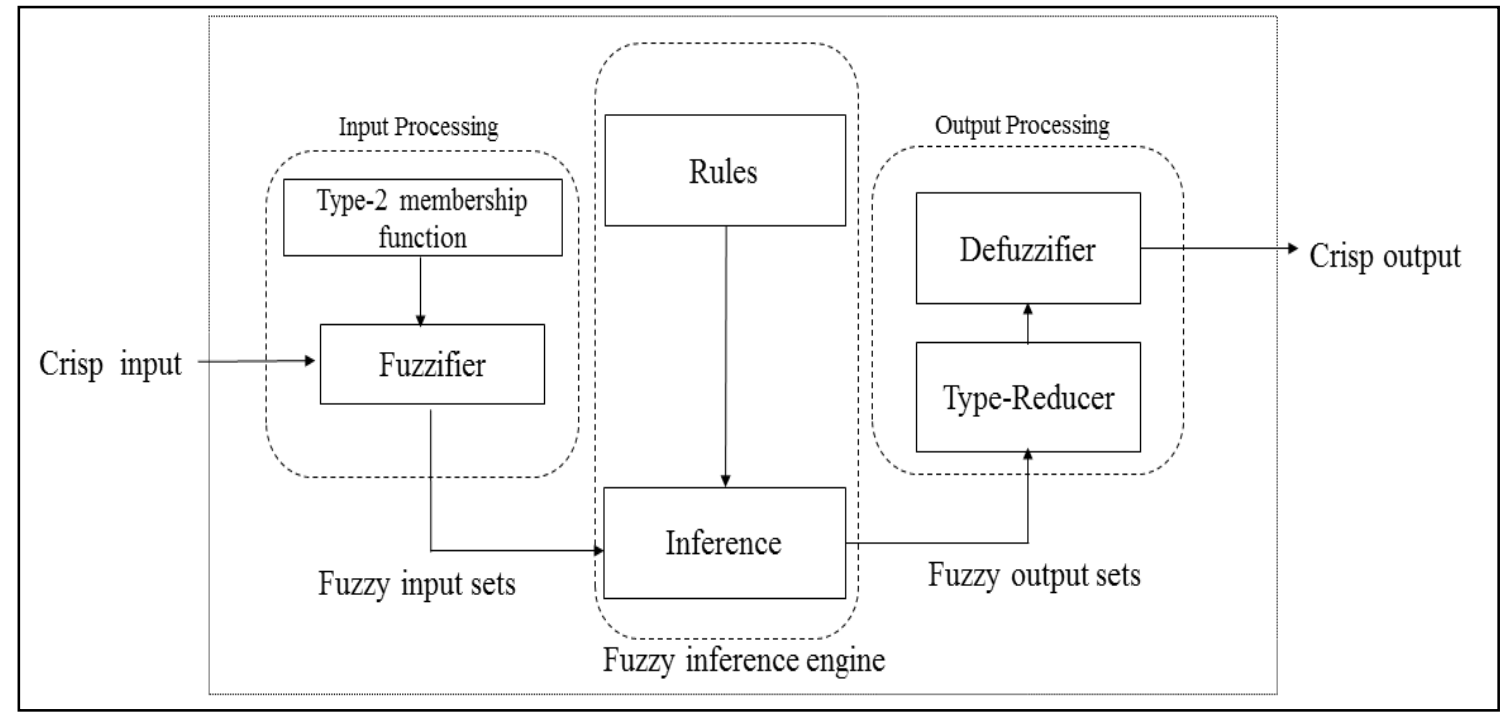

Figure 2 Schematic of proposed IT2FLDS for image fusion applications

The proposed IT2FLDS schematic for image fusion applications mainly contains three different blocks like input processing, FIE and output processing as demonstrated in figure 1. The Sugeno model is utilized to realize the proposed IT2FLDS. Medical data from healthcare solutions act as an IT2FLDS crisp inputs. Input medical data can be denoted as set I. To map medical input data I into fuzzy set $\ddot{F}$, a fuzzifier is utilized with the help of type-2 fuzzy membership functions $\gamma_{\ddot{D}}$. Medical input data $\mathrm{I}$ can be changed into fuzzy values using fuzzifier. The T2F reasoning is applied with the help of Fuzzy inference engine (FIE) on fuzzy sets $\ddot{F}$ to attain fuzzy output set $S$. Here, If ... Then fuzzy rules are responsible for Type-2 fuzzy reasoning in Fuzzy inference engine (FIE). The Output handling block

\subsection{Type-2 fuzzy input set realization:}

T2FS Represents FS whose MF grades are themselves T1FS. A T2FS can be represented as $\ddot{F}$ and evaluated using $M F$ of T2FS $\gamma_{\ddot{F}}(x, y)$ and can be explained as follows: gives crisp outputs $\mathrm{N}$, which rely upon fuzzy output set $\mathrm{S}$ which can be attained using FIE.The Output handling block also contains a type- 2 reducer and defuzzifier blocks. To convert T2FS into T2F1, a Type reducer method KarnikMendel can be utilized. The type reduced output block can be converted into accurate crisp output values with the help of Defuzzifier. Here, we have utilized a wtaver based defuzzification method in our proposed IT2FLDS.

Crisp input set $M$ is represented as the medical data set which is acquired from various healthcare solutions to fuse the multi-model medical color images. In IT2FLDS the Gaussian IT $2 F M F$ are utilized to map $M$ into fuzzy sets.

$$
\begin{array}{r}
\ddot{F}=\left((x, y), \gamma_{\ddot{F}}(x, y)\right) \mid \forall x \in M, \forall y \in T_{x} \\
\quad \subseteq[0,1] \\
\text { Where } x \in M, \quad y \in T_{x} \subseteq[0,1]
\end{array}
$$
and $\mathrm{N} \leq$ $\gamma_{\ddot{F}}(x, y) \leq 1$. In (1), $T_{x}$ shows the codomain at every $x \in M$ of IT $2 F S \ddot{F}$.

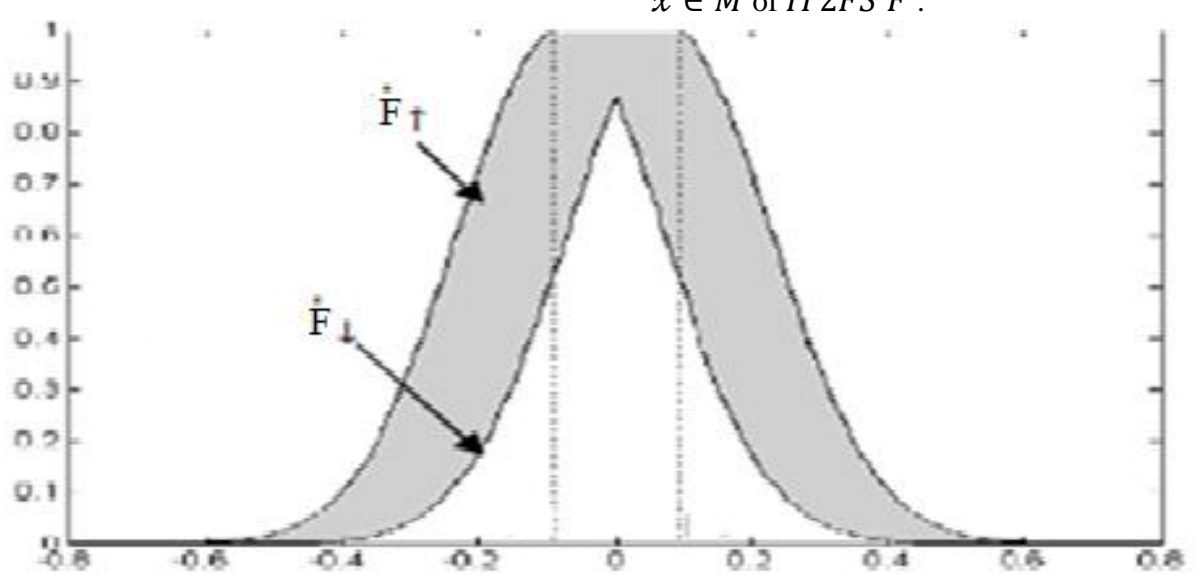

Figure 3 A Gaussian interval type-2 fuzzy membership set $\ddot{F}$.The upper $\dot{\mathcal{E}}_{\uparrow}$ and lower $\dot{\mathcal{E}}_{\downarrow}$ membership functions are also shown.

Here, figure 3 represents a Gaussian interval T2MF set $\ddot{F}$ where $\dot{\mathcal{E}}_{\uparrow}$ shows higher membership functions whereas $\dot{\mathcal{E}}_{\downarrow}$ shows lower membership functions of IT2FLDS. A T2MF is a blurred version of membership function of type 1 [31, 32]. 
Here, type-1 Gaussian function is denoted by a black thick dotted line i.e. $\dot{\varepsilon}_{n}$. The type-1 Gaussian function is presented as $\left(\mathrm{a}_{n 1}, \mathrm{a}_{n 2}, \mathrm{a}_{n 3}, \mathrm{a}_{n 4}\right)$.

Here, figure 2 clearly shows that the Gaussian T2MF contains higher and lower membership function of type-1 Gaussian function i.e. $\dot{\mathcal{E}}_{\uparrow}$ and $\dot{\mathcal{E}}_{\downarrow}$. Then, a IT2 Gaussian function can be denoted as $\ddot{\mathrm{F}}_{n}$ and it is represented in the discourse Universe $\mathrm{M}[33]$ as,

$$
\ddot{\mathrm{F}}_{n}=\left(\dot{\varepsilon}_{\uparrow n}, \dot{\varepsilon}_{\downarrow n}\right) \text {, }
$$

Where $\dot{\varepsilon}_{\uparrow n}$ and $\dot{\varepsilon}_{\downarrow n}$ shows higher and lower TIFS of Gaussian T2MF. FS of Type-2 $\ddot{\mathrm{F}}_{n}$ can be expressed as,

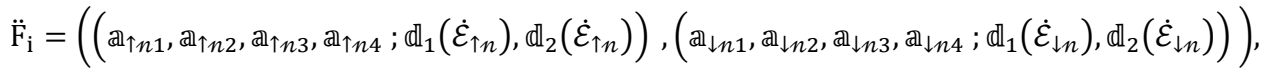

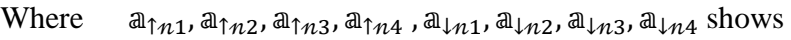
the set $\ddot{\mathrm{F}}_{n}$ reference points. In the interval $1 \leq \mathrm{j} \leq 2$, the membership value of element $\mathbb{a}_{\uparrow n(\mathrm{j}+1)}$ th in set $\dot{\mathcal{E}}_{\uparrow n}$ can be denoted as $\mathbb{d}_{\mathrm{j}}\left(\dot{\varepsilon}_{\uparrow n}\right)$ in (3).Likewise, in the interval $1 \leq \mathrm{j} \leq$ 2 , the membership value of element $\mathrm{a}_{\downarrow n(\mathrm{j}+1)}$ th in set $\dot{\varepsilon}_{\downarrow n}$ can be denoted as $\mathbb{d}_{j}\left(\dot{\varepsilon}_{\downarrow_{n}}\right)$ in (3). In (3) $\mathbb{d}_{1}\left(\dot{\varepsilon}_{\uparrow n}\right) \in[-1,1], \mathbb{d}_{2}\left(\dot{\varepsilon}_{\uparrow n}\right) \in[-1,1], \mathbb{d}_{1}\left(\dot{\varepsilon}_{\downarrow n}\right) \in$ $[-1,1], \mathbb{d}_{2}\left(\dot{\varepsilon}_{\downarrow n}\right) \in[-1,1]$ and $1 \leq x \leq 2$.

\subsubsection{Fuzzifier:}

Here, we discuss about the fuzzifier used in our sugeno model. This block requires a crisp input i.e. medical data $x=\left(x_{1}, \ldots, x_{r}\right)^{G} \in M_{1} \times M_{2} \times \ldots . M_{r} \equiv M$ and which is mapped into input fuzzy set $\ddot{F}_{x}$ for $M$. For any conversion,

\subsubsection{Fuzzy Rules:}

Assume that a fuzzy rule set which consist of $P$ rules defined by the human knowledge. The design of presenting rules in FT 1 and FT2 are same. The fuzzy rules contains $I F \ldots$ and THEN ... segment. Here, the $I F \ldots$ segment is termed as antecedent and THEN ... segment is termed as consequence. membership functions of fuzzy can be used. In the model IT2FLDS, a Gaussian membership function of type-2 is utilized. Typically, in our sugeno model a type- 2 singleton fuzzifier is utilized which explains that the fuzzy input set consists of a single point of non-zero membership type. To take decisions in FIE, the output of fuzzifier i.e. Output fuzzy set $S$

\subsection{Type-2 Sugeno Fuzzy Inference Engine:}

The primary function of Fuzzy Inference Engine $(F I E)$ is represented by the decision making from human knowledge. A decision making mechanism and various sets of fuzzy rules are incorporated in the Fuzzy Inference Engine $(F I E)$. In our model IT2FLDS, a sugeno type Interval Type-2 Fuzzy Inference Engine is utilized.

In our model IT2FLDS, the antecedent and consequence segments are defined using $S$ of $T 2$. Assume that, the linguistic variables $r$ are input which can be expressed as $x_{1} \in M_{1}, \ldots x_{r} \in M_{r}$ and the fuzzy output $c \in N$. Then, the $h^{\text {th }}$ fuzzy rules explained as,

$$
Q^{h}: I F \quad x_{1} \text { is } \ddot{S}_{1}^{h} \text { and } x_{2} \text { is } \ddot{S}_{2}^{h} \text {... and } x_{r} \text { is } \ddot{S}_{R}^{h} \text { THEN } c \text { is } \ddot{V}^{h} h=1,2,3 \ldots P \text {, }
$$

Where $S$ and $V$ shows suitable fuzzy sets for every rule. Numerous rules are introduced to precisely define human knowledge and links which are present between antecedents and consequence. In a rule set $Q$, there are $P$ rules present.

\subsubsection{Sugeno Interval Type-2 Fuzzy Inference}

Engine:

Fuzzy implication method 'prod' is utilized by Fuzzy Inference Engine (FIE) to convert FT2IS into FT2OS. A relationship can be established between fuzzy type-2 input and output sets using $F I E$ by joining rules $Q$. The cases in

$$
Q^{h}: \ddot{S}_{1}^{h} \times \ddot{S}_{2}^{h} \ldots \times \ddot{S}_{R}^{h} \longrightarrow \ddot{V}^{h}=\ddot{K}^{h} \rightarrow \ddot{V}^{h} h=1,2,3, \ldots P
$$

which antecedent are more, join $U$ and meet $\bigcap$ operators can be utilized to provide a single number which shows the actual results of that rule. More than one rule can be employed concurrently. When more than one rule is applied, the results obtained from each rule is combined together to make a resultant type-2 fuzzy output. To make certain each type-2 relation attained from set $Q$ are drawn, union $U$ and intersection $\cap$ computations and sup star structures of type-2 links are utilized. If $\ddot{S}_{1}^{h} \times \ddot{S}_{2}^{h} \ldots \times \ddot{S}_{R}^{h}=\ddot{K}^{h}$ then $h^{\text {th }}$ fuzzy rule is expressed as,

Where, the membership function $\gamma_{Q^{h}}(\boldsymbol{x}, c)=$ $\gamma_{Q^{h}}\left(x_{1}, \ldots, x_{r}, o\right)$ can be expressed using $Q^{h}$. Then, the

$$
\gamma_{Q^{h}}(\boldsymbol{x}, c)=\gamma_{\ddot{K}^{h} \rightarrow \ddot{V}^{h}}(\boldsymbol{x}, c)
$$
$M F \gamma_{Q^{h}}(\boldsymbol{x}, c)$ can be evaluated as,

$$
\gamma_{Q^{h}}(x, c)=\gamma_{\ddot{S}_{1}^{h}}\left(x_{1}\right) \cap \gamma_{\ddot{S}_{2}^{h}}\left(x_{2}\right) \cap \gamma_{\ddot{S}_{3}^{h}}\left(x_{3}\right) \cap \ldots \cap \gamma_{\gamma_{\ddot{S}_{r}^{h}}}\left(x_{r}\right) \cap \gamma_{\ddot{V}^{h}}(\mathrm{c}) \text {. }
$$

By Simplifying (7) we get,

$$
\gamma_{Q^{h}}(\boldsymbol{x}, c)=\left[\prod_{y=1}^{r} \gamma_{\ddot{S}_{y}^{h}}\left(x_{y}\right)\right] \prod \gamma_{\ddot{V} h}(c) .
$$

A $T 2 F S \ddot{K}^{h}$ is utilized to get $r$ inputs of the $h^{\text {th }}$ rule. Then, the membership function $\gamma_{\ddot{K}}$ for a T2FS $\ddot{K}^{h}$ is expressed as,

$$
\gamma_{\ddot{K}_{x}}(\boldsymbol{x})=\gamma_{\ddot{x}_{1}}\left(x_{1}\right) \cap \gamma_{x}\left(x_{2}\right) \ldots \cap \gamma_{\ddot{x}_{r}}\left(x_{r}\right)
$$

$$
\gamma_{\ddot{K}_{x}}(\boldsymbol{x})=\prod_{y=1}^{r} \gamma_{\ddot{x}_{y}}\left(x_{y}\right),
$$

Where fuzzy set labels can be expressed as $\ddot{M}_{y}(y=$ 
$1,2,3, \ldots, r)$ which defines $r$ inputs. The compositions $\ddot{A}^{h}=\ddot{K}_{x} \circ Q^{h}$ are utilized for every set $Q$ in such a way that it can define $T 2 F S$ and evaluated as,

$$
\begin{gathered}
\gamma_{\ddot{A}^{h}}(c)=\gamma_{\ddot{K}_{x} \circ Q^{h}} \\
\gamma_{\ddot{A}^{h}}(c)=\bigcup_{x \in I}\left[\gamma_{\ddot{K}_{x}}(x) \cap \gamma_{Q^{h}}(x, c)\right], \forall c \\
\in N, \forall h=1,2,3 \ldots, P
\end{gathered}
$$

In the proposed model IT2FLDS, T2FSand intersection

$$
\begin{aligned}
& l_{\downarrow}^{h}\left(x^{\prime}\right)=\gamma_{\downarrow \tilde{S}_{1}^{h}}\left(x_{1}^{\prime}\right) * \gamma_{\ddot{J}_{2}^{h}}\left(x_{2}^{\prime}\right) * \gamma_{\ddot{\jmath}_{3}^{h}}\left(x_{3}^{\prime}\right) * \cdots \gamma_{\ddot{l}_{r}^{h}}\left(x_{r}^{\prime}\right) \text {, } \\
& l_{\uparrow}^{h}\left(x^{\prime}\right)=\gamma_{\oplus_{1}^{h}}\left(x_{1}^{\prime}\right) * \gamma_{\ddot{\uparrow}_{2}^{h}}\left(x_{2}^{\prime}\right) * \gamma_{\uparrow_{3}^{h}}\left(x_{3}^{\prime}\right) * \cdots \gamma_{\ddot{\uparrow}_{r}^{h}}\left(x_{r}^{\prime}\right) \text {. }
\end{aligned}
$$

\subsubsection{Output Processing:}

A type-2 fuzzy reducer segment and a defuzzifier segment is present in the IT2FLDS output processing block. The crisp output set $N$ can be attained using the fuzzy output set which can be obtained using fuzzy inference engine $(F I E)$.

\section{i) Type Reducer:}

The output set of Fuzzy Inference Engine can be converted $N_{\mathbb{C O S}}(x)$

$$
\begin{array}{r}
=\int c^{1} \in\left[c_{1}^{h}, c_{e}^{1}\right] \int c^{2} \in\left[c_{2}^{h}, c_{e}^{2}\right] . \int c^{P} \in\left[c_{h}^{P}, c_{e}^{P}\right] \int l^{1} \in\left[l_{\uparrow}^{1} \cdot l_{\uparrow}^{1}\right] . \iint^{P} \\
\in\left[l_{\uparrow}^{P} \cdot l_{\uparrow}^{P}\right] 1 / \frac{\sum_{y=1}^{P} l^{y} c^{y}}{\sum_{y=1}^{P} l^{y}} .
\end{array}
$$

The end points $c_{h}$ and $c_{e}$ related to the type- 2 interval set $\ddot{V}^{b}$ is expressed as wtaver of consequent set $\ddot{V}^{b}$. The wtaver of consequent

$$
A_{\ddot{V} y}=\int \phi_{1} \in O_{c^{1}} \int \phi_{2} \epsilon O_{c^{2}} . \int \phi_{X} \in O_{c^{X}} \quad 1 / \frac{\sum_{y=1}^{X} \phi_{y} c_{y}}{\sum_{y=1}^{X} \phi_{y}}=\left[c_{h}^{y}, c_{e}^{y}\right],
$$

Where $\phi_{y}$ ranges in the interval $[0,1]$ and $\ddot{V}^{y}$ contains $X$ distinct points. The parameters $c_{h}^{y}$ and $l_{h}^{y}$ shows link of $c_{y}$ and $l_{y}$ with $c_{h}$. Likewise, $c_{e}^{y}$ and $l_{e}^{y}$ shows link of $c_{y}$ and $l_{y}$ with $c_{e}$.

Here, $c_{h}^{y}$ and $c_{e}^{y}$ are evaluated with the help of (15) and then utilized to evaluate $N_{\mathbb{C O S}}(x)$ in our proposed model IT2FLDS. IT2FLDS output interval i.e. $c_{h}$ and $c_{e}$ is

\section{ii) Defuzzification}

The proposed IT2FLDS model utilizes wtaver technique for defuzzification process. Using type reduction Karnik-Mendel method an interval set $J_{\mathbb{C o s}}$ is obtained. The crisp outputs can be attained using wtaver technique by averaging $j_{h}$ and $j_{e}$ variables. Then, IT2FLDS Defuzzification can be attained using,

$$
c(x)=\left(c_{h}+c_{e}\right) 0.5 .
$$

Crisp outputs attained after defuzzification process helps to determine accurate fusion results for medical images. In this way, an efficient fusion of medical images is evaluate using IT2FLDS

\section{PERFORMANCE EVALUATION:}

This section determines about the performance of the proposed fusion technique based on IT2FLDS. Here, four different set of medical color images are taken to evaluate the performance of the system. MATLAB 12B is utilized to carried out each experiment. IT $2 F L D S$ can be implemented described as,

$$
\begin{gathered}
c_{h}=\left(\sum_{x=1}^{P} l_{h}^{y} c_{h}^{y} / \sum_{x=1}^{P} l_{h}^{y}\right), \\
c_{e}=\left(\sum_{x=1}^{P} l_{e}^{y} c_{e}^{y} / \sum_{x=1}^{P} l_{e}^{y}\right),
\end{gathered}
$$

The $\mathbb{C} \mathbb{S}$ reduction method output is defuzzified to attain crisp outputs.

using $T 2 F$ toolbox. Fuzzy Inference Engine $(F I E)$, membership functions and fuzzy rules introduced to evaluate the efficiency of the proposed model. IT2FLDS can control uncertainities in a much efficient way which is verified with initial experimental outcomes. Moreover, medical image fusion technique is handled by processing of medical data from heathcare solutions.Our proposed image fusion technique is emloyed on clinically attained mutiple color medical images. The color medical image dataset utilized in our model are of various types. This dataset is an open source dataset. The efficiency of proposed image fusion technique can be determined by conducting various experiments on different color images of multiple modalities.

\subsection{Color Image fusion using our proposed model:}

Here, in this section an effective color image fusion technique is introduced for color sensor images. Let that PET and SPECT images are color images which are assumed to a pseudo-color. Here, the gray and pseudo color images are 
fused together into a single image to provide more detailed biological information. Moreover, RGB color space consists of similar resolution for all the three Red, Green and Blue color. All the three channels of RGB color space, work independently based on fusion modalities which is used to decrease the blurriness in the fused images and get more details. Moreover, High luminance values can demonstrate a color image in a much effective manner by segregating the brightness and color info. In our model, a YUV color map is used which is type of color image pipeline. The YUV map is motivated by Opponent-process theory which is a physiological model which defines variety of behaviours like colour vision. Moreover, this technique is utilized to encode the color images or videos achromatic and chromatic colors which are available in all the three dimensions. Moreover, in YUV color space, $\mathrm{Y}$ component describes about the luminance/ brightness of an image. Similarly, the U and V components describes about the color itself. As demonstrated in figure 1, the $\mathrm{U}$ and $\mathrm{V}$ components of YUV color space can be discarded to attain a gray scale-image. The intensity of a color image can be enhance without disturbing the color due to disconnection between gray scale and chrominance signals. This shows YUV is very much appropriate for image fusion.

This image fusion technique gives more contrast increment than any other state-of-art techniques due to its complete color utilization with the help of Opponent-process theory in human perception. In our proposed image fusion technique, the main idea is to convert the input color image from RGB to YUV color space. Then, both the achromatic channel Y of the input color image and input gray scale image is fused together using the proposed image fusion technique to get high intensity of a color image. Finally, the YUV color space is again converted into RGB color space. In this manner, a final fused image is attained with the help of our proposed image fusion technique as demonstrated in figure 1 .

\subsection{Comparative Study:}

In this subsection, we discuss about the performance comparison of our proposed image fusion technique with various state-of-art-techniques using interval type-2 fuzzy logic decision system (IT2FLDS). The comparison of our proposed image fusion technique is demonstrated in table 1 in terms of quantitative matrices such as Mutual Information $(\mathbb{M I})$, Standard Deviation (STD) and Feature Point Similarity Measure $\left(\mathbb{Q}^{\mathbb{A B} / \mathbb{f}}\right)$ for four different group of color medical images. Here, the range of Feature Point Similarity
Measure $\left(\mathbb{Q}^{\mathbb{A B} / \mathbb{F}}\right)$ is 0 to 1 whereas Mutual Information $(\mathbb{M I})$ and Standard Deviation (STD)are largest value matrices. Here, the higher value of $\mathbb{M I I}$ than any other conventional state-of-art-techniques represents the dominance of our proposed image fusion technique. The quantitative outcomes demonstrates superiority and robustness of our proposed image fusion technique in terms of detailed extracted information from input images and spatial consistency of fused output images which is much sharper than any other state-of-art-techniques. The fused image obtained using our proposed image fusion technique consists of more detailed data, provide larger structural similarity and correlation to the source image in contrast to conventional techniques as demonstrated in table 1 .

Various experiments are performed on different multimodal medical color images taken from the standard medical color test images to verify the accountability of the proposed image fusion technique. The experimental results are carried out by considering four different medical image group of various combinations such as MRI/PET, MRI/SPECT demonstrated in figure 4. In figure 4(a) and 4(b), MRI/PET fusion image group is considered in which one source image is of MRI type and other is of PET scan type of brain. The fused image consist of metabolic information from PET input image and anatomical structural information from MRI images, which is better than any other state-of-art-techniques in terms of structural information and visual quality. The figure 4(c) and 4(d), MRI/ SPECT fusion image group is considered in which one source image is of MRI type and other is of SPECT scan type. The fused image consist of brain structures information of non-functional areas from MRI input image and color structural information of SPECT images of functional areas. The evaluated quantitative matrices are described using input gray scale images of MRI/CT type and color images of PET/SPECT type.

The conventional state-of-art-techniques such as NSCT, Scheme-1[34] and Scheme-2 [35] are utilized to compare the proposed image fusion technique and verify its superiority than any other state-of-art-techniques demonstrated in table 1. Experimental results verifies superiority of proposed image fusion technique in terms of visual and statistical and structural information attained in the fused image based on interval type- 2 fuzzy logic decision system and fused images are more clearer and contained higher contrast.

Table 1 Evaluation indices for fused medical images

\begin{tabular}{|l|c|l|l|l|l|l|}
\hline Group & INDICES & NSCT & Scheme-1[34] & Scheme-2[35] & NSCT T2FLDS & PROPOSED \\
\hline \multirow{2}{*}{$\begin{array}{l}\text { Group1 } \\
\text { MRI/PET }\end{array}$} & $\mathbb{M I I}$ & 2.32 & 2.44 & 2.91 & 2.96 & $\mathbf{4 . 1 0 3}$ \\
\cline { 2 - 7 } & $\mathbb{Q}^{\mathrm{AII} / \mathbb{I}}$ & 0.59 & 0.61 & 0.63 & 0.65 & $\mathbf{0 . 6 9 3}$ \\
\cline { 2 - 7 } & $\mathbb{S T I D}$ & 67.9 & 70.2 & 81.0 & 81.7 & $\mathbf{8 5 . 0 9}$ \\
\hline \multirow{3}{*}{$\begin{array}{l}\text { Group2 } \\
\text { MRI/PET }\end{array}$} & $\mathbb{M I I}$ & 2.54 & 2.48 & 2.90 & 3.11 & $\mathbf{3 . 6 5 6}$ \\
\cline { 2 - 7 } & $\mathbb{Q}^{\mathrm{AII} / \mathbb{I}}$ & 0.52 & 0.50 & 0.53 & 0.55 & $\mathbf{0 . 5 1 3}$ \\
\cline { 2 - 7 } & $\mathbb{S T I D}$ & 53.2 & 54.0 & 62.1 & 62.7 & $\mathbf{6 0 . 1 2 7}$ \\
\hline \multirow{2}{*}{$\begin{array}{l}\text { Group3 } \\
\text { MRI/SPECT }\end{array}$} & $\mathbb{M I}$ & 2.21 & 2.08 & 2.38 & 2.51 & $\mathbf{3 . 0 6 3}$ \\
\cline { 2 - 7 } & $\mathbb{Q}^{\mathrm{AB} / \mathbb{I}}$ & 0.52 & 0.50 & 0.524 & 0.525 & $\mathbf{0 . 4 6 2 2}$ \\
\cline { 2 - 6 } & $\mathbb{S T I D}$ & 47.1 & 47.3 & 52.1 & 53.4 & $\mathbf{5 3 . 9 6}$ \\
\hline Group4 & $\mathbb{M I I}$ & 1.68 & 1.53 & 2.46 & 2.49 & $\mathbf{4 . 6 4}$ \\
\hline
\end{tabular}




\begin{tabular}{|l|l|l|l|l|l|l|}
\hline MRI/SPECT & $\mathbb{Q}^{\mathbb{A B} / \mathbb{f}}$ & 0.62 & 0.52 & 0.64 & 0.64 & $\mathbf{0 . 7 4 3}$ \\
\cline { 2 - 7 } & $\mathbb{S T T D}$ & 49.1 & 60.0 & 68.2 & 68.2 & $\mathbf{6 6 . 1 3}$ \\
\hline
\end{tabular}

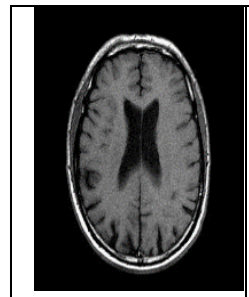

(A1)

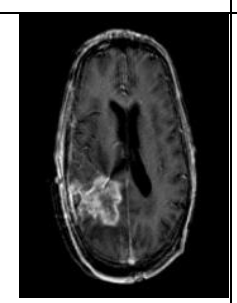

(A2)



(A3)

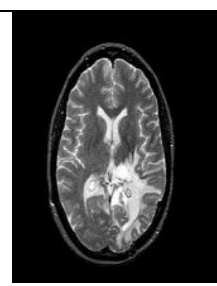

(A4)

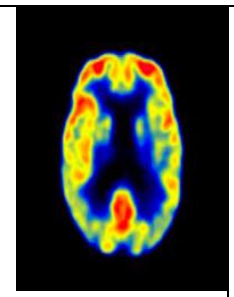

(B1)



(B2)

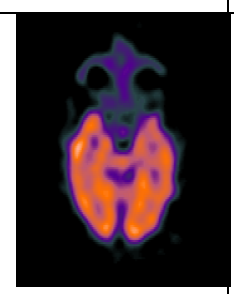

(B3)

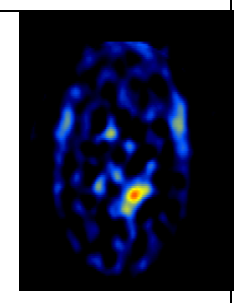

(B4)

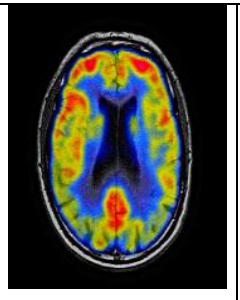

(C1)

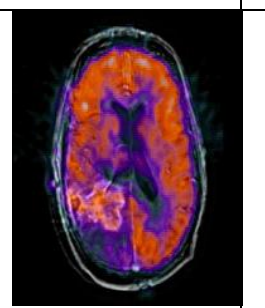

(C2)

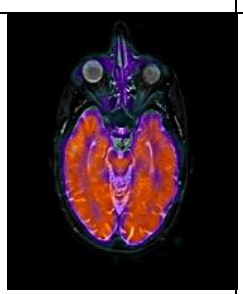

(C3)

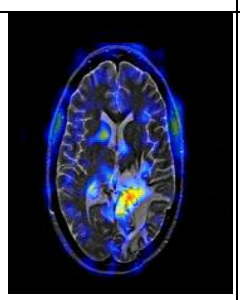

(C4)

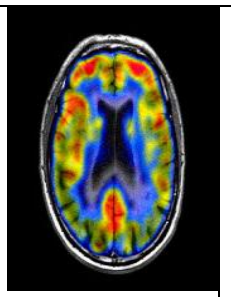

(D1)

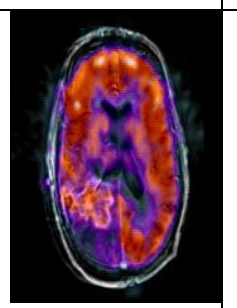

(D2)

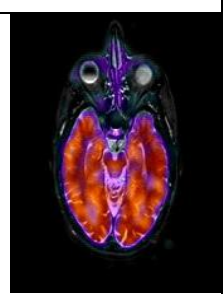

(D3)

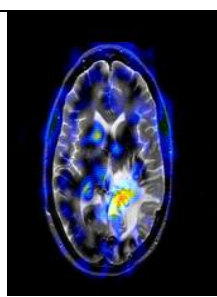

(D4)

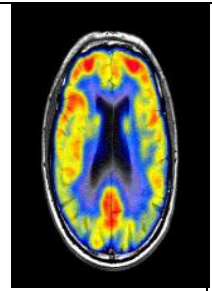

(E1)
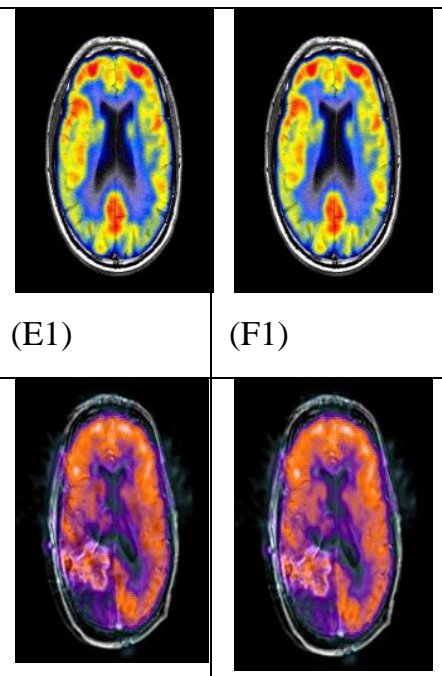

(E2)

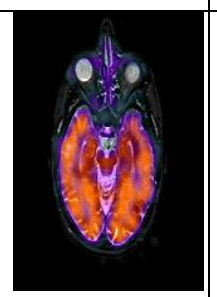

(E3)

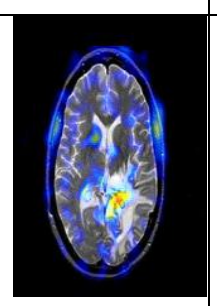

(E4)
(F1)

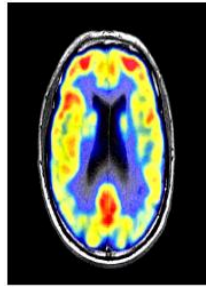

(G1)

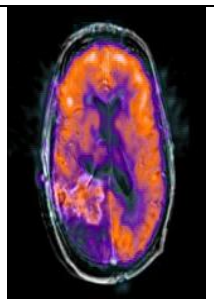

(F2)

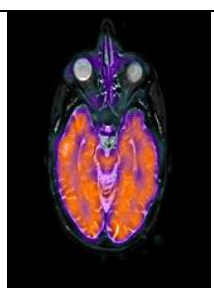

(F3)

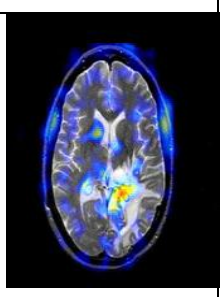

(F4)

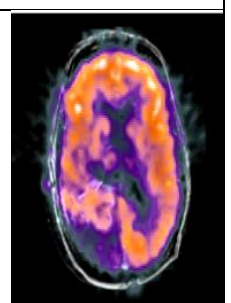

(G2)

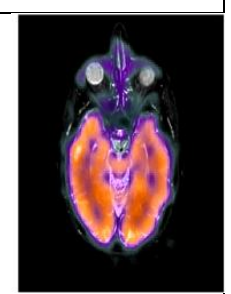

(G3)



(G4)

Figure 4 Group 1 through Group 4 source multimodal medical images and results: (A1)-(A4) MRI images. (B1) and (B2) PET images. (B3) and (B4) SPECT images. (C1) - (C4) by NSCT method, (D1)-(D4) by scheme [34] method, (E1)-(E4) by scheme [35] method, (F1)-(F4) by NSCT+IT2FLDS method, (G1)-(G4) by proposed method.

\section{Graphical Representation:}

In this subsection, the graphical representation for the performance of the proposed image fusion technique with conventional state-of-art-techniques is shown. Here, figure 5 demonstrates comparison of mutual information using the proposed image fusion technique based on IT2FLDS with existing state-of-art-techniques for group 1 to group 4 images.
Here, figure 6 demonstrates comparison of Feature Point Similarity Measure using the proposed image fusion technique based on IT2FLDS with existing state-of-arttechniques for group 1 to group 4 images. Here, figure 7 demonstrates comparison of standard deviation using the proposed image fusion technique based on IT2FLDS with existing state-of-art-techniques for group 1 to group 4 images. 




Figure 5 comparison of mutual information with existing state-of-art-techniques using proposed image fusion technique

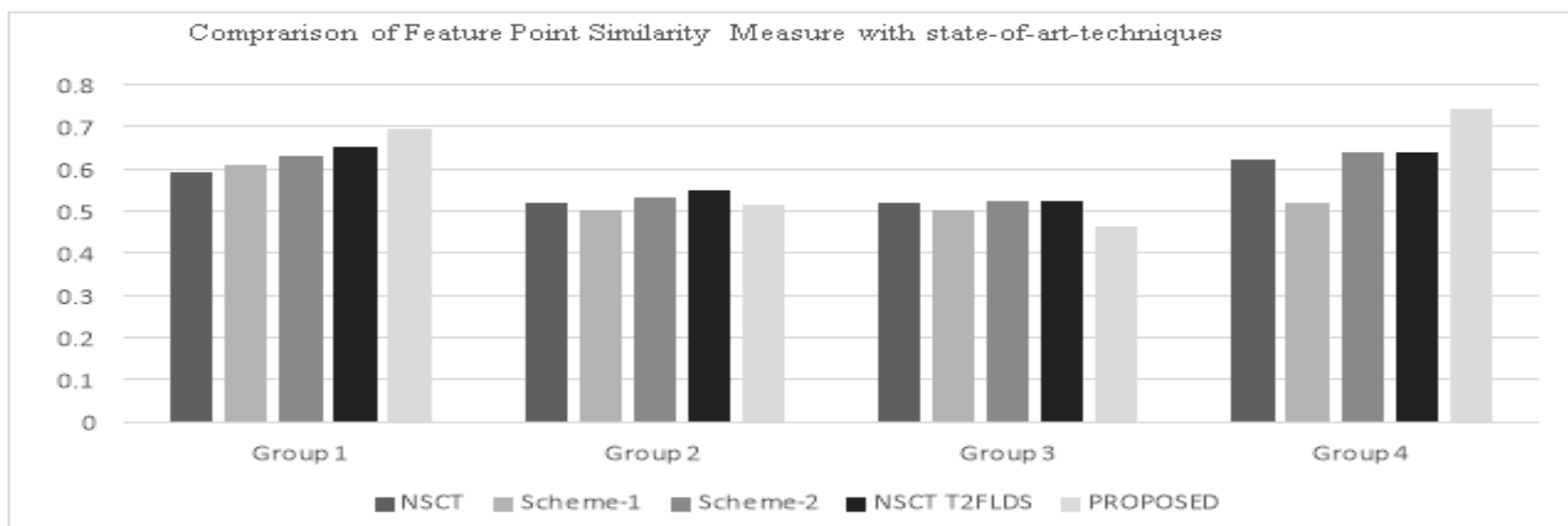

Figure 6 comparison of feature point similarity measure with existing state-of-art-techniques using proposed image fusion technique

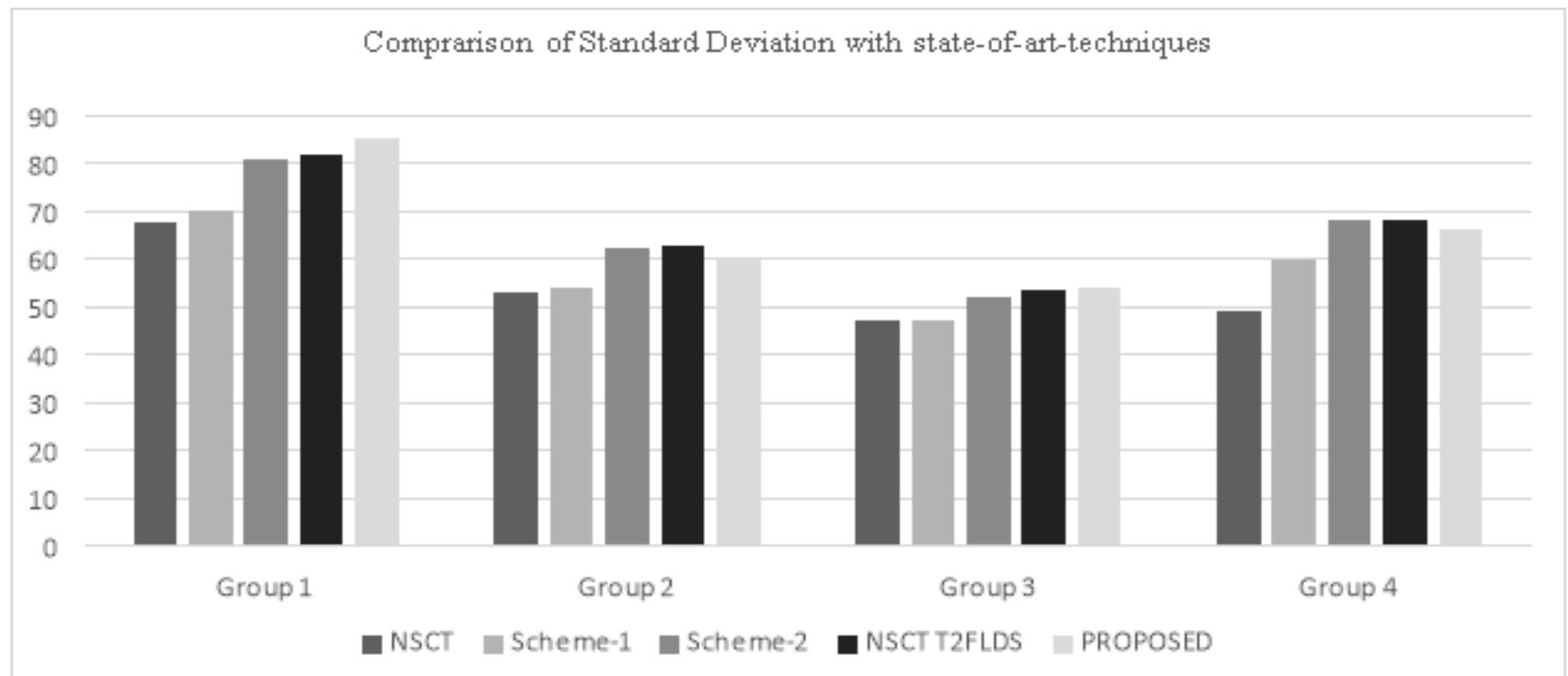

Figure 7 comparison of standard deviation with existing state-of-art-techniques using proposed image fusion technique

\section{CONCLUSION}

The significance of IT2FLDS is very essential in medical applications. Moreover, Medical imaging and information processing technologies has taken drastic growth to counter the drawbacks of clinical medical image assessment. To eliminate the drawbacks of medical imaging sensor applications, the Multimodal medical color image fusion 
technique can be an essential method. Therefore, here, we present an image fusion technique to control non-linear uncertainties and provide stability based on IT2FLDS for multi-modal medical color images based on Sugeno model. This image fusion technique helps in clinical diagnosis of various diseases. An efficient modelling is presented for image fusion model based on IT2FLDS. The efficiency of proposed image fusion technique can be determined by conducting various experiments on different color images of multiple modalities IT2FLDS helps to eliminate uncertainties and The fused image obtained using our proposed image fusion technique consists of more detailed data, provide larger structural similarity and correlation to the source image in contrast to conventional techniques. Experimental results verifies superiority of proposed image fusion technique in terms of visual and statistical and structural information attained in the fused image based on IT2FLDS and fused images are more clearer and contained higher contrast. The quantitative outcomes demonstrates superiority and robustness of our proposed fusion technique in terms of mutual information, standard deviation and Feature Point Similarity Measure.

\section{REFERENCES}

[1] A. P. James and B. V. Dasarathy, "Medical image fusion: A survey of the state of the art," In! Fusion, vol. 19, pp. 4-19, 2014

[2] C. 1. Price and K. 1. Friston, "Functional imaging studies of neuropsychological patients: applications and limitations," Neurocase, vol. 8, no. 5, pp. 345-354, 2002.

[3] Yu Liua, Xun Chena, Hu Penga, et al. Multi-focus image fusion with a deep convolutional neural network[J].Information Fusion, 2017,36:191-207.

[4] Lei Wang, Bin Li, Lian-fang Tian. Multi-modal medical image fusion using the inter-scale and intra-scale dependencies between image shift-invariant shearlet coefficients[J]. Information Fusion,2014,19:20-28.

[5] M.A. Rahman, S. Liu, S. Lin, C. Wong, G. Jiang, N. Kwok, Image contrast en-hancement for brightness preservation based on dynamic stretching, Int. J. Image Proces. (IJIP) 9(4) (2015) 241.

[6] M.A. Rahman, S. Lin, C. Wong, G. Jiang, S. Liu, N. Kwok, Efficient colour image compression using fusion approach, Imaging Sci. J. (2015),

[7] A. Goshtasby, S. Nikolov, Image fusion: advances in the state of the art, Information Fusion 8 (2) (2007) 114-118.

[8] A. James, B. Dasarathy, Medical image fusion: A survey of the state of the art, Information Fusion 19 (2014) 419. 1,2

[9] A. Wang, H. Sun, Y. Guan, The application of wavelet transform to multimodality medical image fusion, in: IEEE International Conference on Networking, Sensing and Control, 2006, pp. 270-274. 1, 3

[10] Q. Miao, C. Shi, P. Xu, M. Yang, Y. Shi, A novel algorithm of image fusion using shearlets, Optics Communications 284 (6) (2011) 1540-1547.

[11] A. James, S. Thiruvenkadam, J. Paul, M. Braun, Special issue on medical image computing and systems, Information Fusion 19 (2014) 2-3. 1, 2

[12] M.A. Musen, B. Middleton, R.A. Greenes, "Clinical decision-support systems”, Biomed. Inform. pp. 643-
674, 2014.

[13] M. F. Akay, "Support vector machines combined with feature selection for breast cancer diagnosis". Expert systems with applications, vol. 36, no. 2, pp. 3240-3247, 2009.

[14] M. Pota, M. Esposito, G. De Pietro, "Fuzzy partitioning for clinical DSSs using statistical information transformed into possibility-based knowledge". Knowledge-Based Systems, vol. 67, pp. 1-15, 2014.

[15] L. Zadeh, "Fuzzy sets", Information Control, vol. 8, 1965, pp.338-353.

[16] T. Ishibuchi, H.; Yamamoto, "Rule weight specification in fuzzy rule-based classification systems," IEEE Transactions on Fuzzy Systems, vol. 13, no. 4, pp. 428435, 2005.

[17] J. Roubos, M. Setnes, and J. Abonyi, Learning fuzzy classification rules from data, 2000.

[18] J. M. Mendel: Uncertain Rule-Based Fuzzy Logic Systems: Introduction and New Directions. Upper Saddle River, NJ Prentice-Hall, 2001.

[19] "Applications of type-2 fuzzy logic systems: Handling the uncertainty associated with surveys," presented at FUZZ-IEEE Conf., Seoul, Korea, Aug. 1999.

[20] K. C. Wu, "Fuzzy interval control of mobile robots," Comput. Elect. Eng., vol. 22, no. 3, pp. 211-229, 1996.

[21] A. Ross and A. K. Jain, "Multimodal biometrics: An overview," Signal Processing Conference, 2004 12th European, Vienna, 2004, pp. 1221-1224.

[22] J. Yang, Y. Wu, Y. Wang and Y. Xiong, "A novel fusion technique for CT and MRI medical image based on NSST," 2016 Chinese Control and Decision Conference (CCDC), Yinchuan, 2016, pp. 4367-4372.

[23] C. K. Chaitanya, G. S. Reddy, V. Bhavana and G. S. C. Varma, "PET and MRI medical image fusion using STDCT and STSVD," 2017 International Conference on Computer Communication and Informatics (ICCCI), Coimbatore, 2017, pp. 1-4.

[24] M. S. Dilmaghani, S. Daneshvar and M. Dousty, "A new MRI and PET image fusion algorithm based on BEMD and IHS methods," 2017 Iranian Conference on Electrical Engineering (ICEE), Tehran, 2017, pp. 118121.

[25] Ye Liu, Yang Wang, Lisheng Wang and Shaoli Song, "Cerebral ASL, SPECT and MRI image registration, enhancing fusion and joint analysis system," 2016 IEEE Advanced Information Management, Communicates, Electronic and Automation Control Conference (IMCEC), Xi'an, 2016, pp. 70-74.

[26] V. Bhavana and H. K. Krishnappa, "Fusion of MRI and PET images using DWT and adaptive histogram equalization," 2016 International Conference on Communication and Signal Processing (ICCSP), Melmaruvathur, 2016, pp. 0795-0798.

[27] S. Qi et al., "Multimodal Fusion With Reference: Searching for Joint Neuromarkers of Working Memory Deficits in Schizophrenia," in IEEE Transactions on Medical Imaging, vol. 37, no. 1, pp. 93-105, Jan. 2018.

[28] Haddadpour M, Daneshavar S, Seyedarabi H. PET and 
MRI image fusion based on combination of Hilbert transform (2-D HT) and IHS method. Biomed J 2017.

[29] H. Liu et al., "Label fusion method based on sparse patch representation for the brain MRI image segmentation," in IET Image Processing, vol. 11, no. 7, pp. 502-511, 7 2017.

[30] J. R. Raol, and S.K. Kashyap, "Decision fusion using fuzzy logic type 1 in two aviation scenarios", Journal of Aerospace Sciences and Technologies, Vol. 65, No. 3, pp. 273-286, 2013.

[31] D. Wu and W. W. Tan, "A simplified architecture for type-2 FLSs and its application to nonlinear control," in Proc. IEEE Conf. on Cybernetics and Intelligent
Systems, Singapore, Dec. 2004, pp. 485-490.

[32] O. Castillo and P. Melin, "Recent Advances in Interval Type-2 Fuzzy Systems", 2012, Springer

[33] J. M. Mendel and R. I. B. John, "Type-2 fuzzy sets made simple," in IEEE Transactions on Fuzzy Systems, vol. 10, no. 2, pp. 117-127, Apr 2002.

[34] G. Bhatnagar, Q. M. J. Wu, and Z. Liu, "Directive contrast based mul-timodal medical image fusion in NSCT domain," IEEE Trans. Multime-dia, vol. 15, no. 5, pp. 1014-1024, 2013.

[35] D. S, and K. MK, "A Neuro-Fuzzy Approach for Medical Image Fu-sion,” IEEE Trans. Biomed. Eng., vol. 60, no. 12, pp. 3347-3353, 2013. 